09

\title{
Трехмерные предельно короткие оптические импульсы Эйри в фотонном кристалле с углеродными нанотрубками
}

\author{
(C) М.Б. Белоненко, Е.И. Мостовая \\ Волгоградский государственный университет, \\ 400062 Волгоград, Россия \\ e-mail: ksanchez@bk.ru
}

Поступила в редакцию 10.12.2017 г.

В окончательной редакции 02.12.2018 г.

Принята к публикации 28.12.2018 г.

Рассмотрена задача динамики распространения трехмерных оптических импульсов (световых пуль) Эйри в фотонном кристалле с углеродными нанотрубками. Численно показано, что предполагаемый тип импульса демонстрирует устойчивое и стабильное распространение.

DOI: $10.21883 /$ OS.2019.05.47653.283-18

\section{Введение}

Фотонными кристаллами принято называть среды, у которых диэлектрическая проницаемость периодически меняется в пространстве с периодом, допускающим брэгговскую дифракцию света. Свет, падающий на такую среду, будет отражен либо преломлен на каждой диэлектрической поверхности, и последующая интерференция может быть либо конструктивной, либо деструктивной в зависимости от длины волны. Распространение света с определенным диапазоном энергий может быть запрещено внутри среды, и таким образом создается „фотонная запрещенная зона“ (Ф33). Запрещенная энергетическая зона в фотонном кристалле изменяется в зависимости от угла падения. Только тогда, когда фотоны могут быть полностью отражены от материала при любых углах падения, Ф3З описывается как „полная“ запрещенная зона. Ф33, которые запрещают распространение света только в некоторых направлениях, представляются как „неполные“ зоны [1].

Другим элементом, обладающим не менее увлекательными свойствами, являются углеродные нанотрубки (УНТ). УНТ широко используются в нелинейной оптике благодаря своим уникальным свойствам. Многих исследователей также привлекает простота их строения и бездефектность, что немаловажно для исследований распространения оптических импульсов, для разработки оптических приборов на их основе, но основным и немаловажным качеством, которым обладают УНТ это возможность их использования в качестве среды для образования световых пуль. В подтверждение этому можно сослаться на работы [2-7].

Также было рассмотрено распространение импульсов в однородной и неоднородной средах УНТ. Однородная среда УНТ не позволяет управлять скоростью импульса, но если скорость распространения световых пуль определяется показателем преломления и не может варьироваться в широких пределах, можно провести дополнительную модуляцию показателя преломления, что способствует образованию неоднородной среды (более подробно см. $[8,9])$. В случае брэгговской среды скорость распространения световых пуль будет определяться как периодом, так и глубиной модуляции показателя преломления за счет частичного отражения и последующей интерференции световых пуль. В этом случае также теоретически возможно контролировать скорость распространения оптических пуль [10]. Что касается фотонных кристаллов, то распространение световых пуль в этой среде будет аналогично распространению в брэгговской среде, но вследствие того, что период модуляции показателя преломления меньше, то и ожидаемые эффекты при той же длине распространения должны быть больше.

Исходя из вышесказанного была предложена трехмерная модель распространения световых импульсов в фотонном кристалле с УНТ. В качестве исходных импульсов были выбраны импульсы Эйри, которые обладают минимальным дисперсионным расплыванием [11].

\section{Основные уравнения}

Рассмотрим распространение предельно коротких оптических импульсов в среде УНТ, помещенных в фотонный кристалл, причем электрическое поле направлено вдоль оси нанотрубок. Основные уравнения для этого случая были получены в работе [12] и будут приведены здесь для полноты изложения.

Так, для гамильтониана системы электронов в виде

$$
H=\gamma \sum_{j \Delta \sigma} a_{j \sigma}^{+} a_{j+\Delta \sigma}+\text { h.c. }
$$

где $a_{j \sigma}^{+}, a_{j \sigma}-$ операторы рождения и уничтожения электронов на узле $j$ со спином $\sigma, \gamma-$ интеграл перескока, определяемый перекрытием волновых функций электронов в соседних узлах, соответствующий 
электронный спектр имеет вид [12]

$$
\varepsilon_{s}(\mathbf{p})= \pm \gamma \sqrt{1+4 \cos (a \mathbf{p}) \cos (\pi s / m)+4 \cos ^{2}(\pi s / m)}
$$

где $s=1,2, \ldots, m$, нанотрубка имеет тип $(m, 0)$, $\gamma \approx 2.7 \mathrm{eV}, a=3 b / 2 \hbar, b=0.142 \mathrm{~nm}$ - расстояние между соседними атомами углерода.

Уравнения Максвелла в цилиндрической системе координат можно записать в виде [13]

$$
\frac{\partial^{2} \mathbf{E}}{\partial z^{2}}+\frac{1}{r} \frac{\partial}{\partial r}\left(r \frac{\partial \mathbf{E}}{\partial r}\right)-\frac{1}{c^{2}} \frac{\partial^{2} \mathbf{E}}{\partial t^{2}}+\frac{4 \pi}{c} \frac{\partial \mathbf{j}}{\partial t}=0,
$$

где $\mathbf{E}$ - электрическое поле световой волны, $\mathbf{j}$ - плотность электрического тока, $t-$ время, $c-$ скорость света в среде. Учет калибровки

$$
\mathbf{E}=-\frac{1}{c} \frac{\partial \mathbf{A}}{\partial t}
$$

дает

$$
\frac{\partial^{2} \mathbf{A}}{\partial z^{2}}+\frac{1}{r} \frac{\partial}{\partial r}\left(r \frac{\partial \mathbf{A}}{\partial r}\right)-\frac{1}{c^{2}} \frac{\partial^{2} \mathbf{A}}{\partial t^{2}}+\frac{4 \pi}{c} \mathbf{j}=0 .
$$

Вектор-потенциал А считается имеющим вид $\mathbf{A}=(0, A(z, r, t), 0)$. Плотность тока $\mathbf{j}$ соответственно имеет вид $\mathbf{j}=(0, j(z, r, t), 0)$. Выражение для плотности тока было неоднократно получено $[3,7,14]$ и имеет вид

$$
\begin{gathered}
j=-e n_{0} \sum_{k} B_{k} \sin \left(\frac{k e}{c} A(t)\right), \\
B_{k}=\sum_{s=1}^{m} \int_{-\pi / a}^{\pi / a} d p A_{k s} \cos (k p) \frac{\exp \left(-\beta \varepsilon_{s}(p)\right)}{1+\exp \left(-\beta \varepsilon_{s}(p)\right)}, \\
v_{s}(p)=\frac{\partial \varepsilon_{s}(p)}{\partial p}, \quad A_{k s}=\int_{-\pi / a}^{\pi / a} v_{s}(p) \sin (k p) d p,
\end{gathered}
$$

где $n_{0}$ - концентрация равновесных электронов в УНТ, $e$ - заряд электрона, $\beta=1 / k T$.

Учитывая все вышесказанное, уравнение (4) после перехода к безразмерным величинам может быть представлено в виде

$$
\frac{\partial^{2} \mathbf{A}}{\partial z^{2}}+\frac{1}{r} \frac{\partial}{\partial r}\left(r \frac{\partial \mathbf{A}}{\partial r}\right)+\frac{4 \pi}{c} \mathbf{j}-\frac{m(z, r)}{c^{2}} \frac{\partial^{2} \mathbf{A}}{\partial t^{2}}=0
$$

где $\mathbf{A}$ - вектор-потенциал, $t-$ время, $c-$ скорость света в среде. В (6) введен коэффициент, который учитывает распределение показателя преломления в пространстве $n(z, r)$, что и определяет фотонный кристалл. Далее в численных расчетах это распределение будет задано в виде $n(z, r)=1+\alpha \cos (2 \pi z / \chi)$, где $\alpha$ задает глубину модуляции нелинейности, а $\chi-$ период модуляции. Отметим, что в настоящей работе рассматривается модуляция только вдоль оси $z$.
Здесь важно отметить следующий момент. В силу неоднородности поля вдоль некоторой оси (например, поле направлено и неоднородно вдоль оси $z$ ) ток также неоднороден. Неоднородность тока обусловливает накопление заряда в некоторой области, которое можно оценить из закона сохранения заряда

$$
\begin{gathered}
\frac{d \rho}{d t}+\frac{d j}{d z}=0, \\
\rho \propto \tau \frac{j}{l_{z}} .
\end{gathered}
$$

Здесь $\rho-$ плотность заряда, $j-$ плотность тока вдоль оси $z, \tau$ - длительность импульса электрического поля, $l_{z}$ - характерная длина, на которой изменяется электрическое поле импульса вдоль оси $z$.

Формула (7) позволяет заключить, что существенное влияние на накопленный заряд оказывает длительность предельно короткого импульса. Сделанные оценки, согласно которым накопленный заряд составляет примерно $1-2 \%$ от заряда, дающего вклад в ток, позволяют пренебречь эффектом накопления заряда для фемтосекундных импульсов. Это подтверждают и численные эксперименты для случая УНТ и импульса длительностью в десятки фемтосекунд [15].

Отметим, что уравнение (6) является обобщением широко известного уравнения Синус-Гордона. Также важно подчеркнуть, что в отличие от работы [7] в случае фотонного кристалла имеется важное отличие. А именно в случае фотонного кристалла период модуляции показателя преломления уже сравним с характерным размером области локализации предельно короткого оптического импульса, и необходимо учитывать это обстоятельство.

Исследуемое уравнение решалось численно. Межзонный ток полагался равным нулю. То есть считалось, что спектр световой пули лежит выше видимой части спектра и максимальная частота колебаний в спектре пули лежит в ближней инфракрасной области. Шаг по времени и координате определялся из условий стабильности и уменьшался до тех пор, пока решение не изменялось только в восьмом знаке.

Начальное условие для вектора-потенциала электрического поля световой пули выбиралось в виде

$$
\begin{gathered}
G(x)=\int_{x}^{\infty} \operatorname{Ai}(y) d y . \\
A(z, r, 0)=Q G\left(\frac{z-z_{0}}{\gamma_{z}}+\kappa\left(\frac{z-z_{0}}{\gamma_{z}}\right)^{2}\right) \\
\frac{d A(r, z, 0)}{d t^{\prime}=}=\left.\frac{d G\left(\frac{z-z_{0}-u t}{\gamma_{z}}+\kappa\left(\frac{z-z_{0}-u t}{\gamma_{z}}\right)^{2}\right)}{d t}\right|_{t^{\prime}=0} \\
\times J_{0}\left(\frac{r}{\gamma_{r}}\right) \exp \left(-\frac{r}{\gamma}\right) .
\end{gathered}
$$



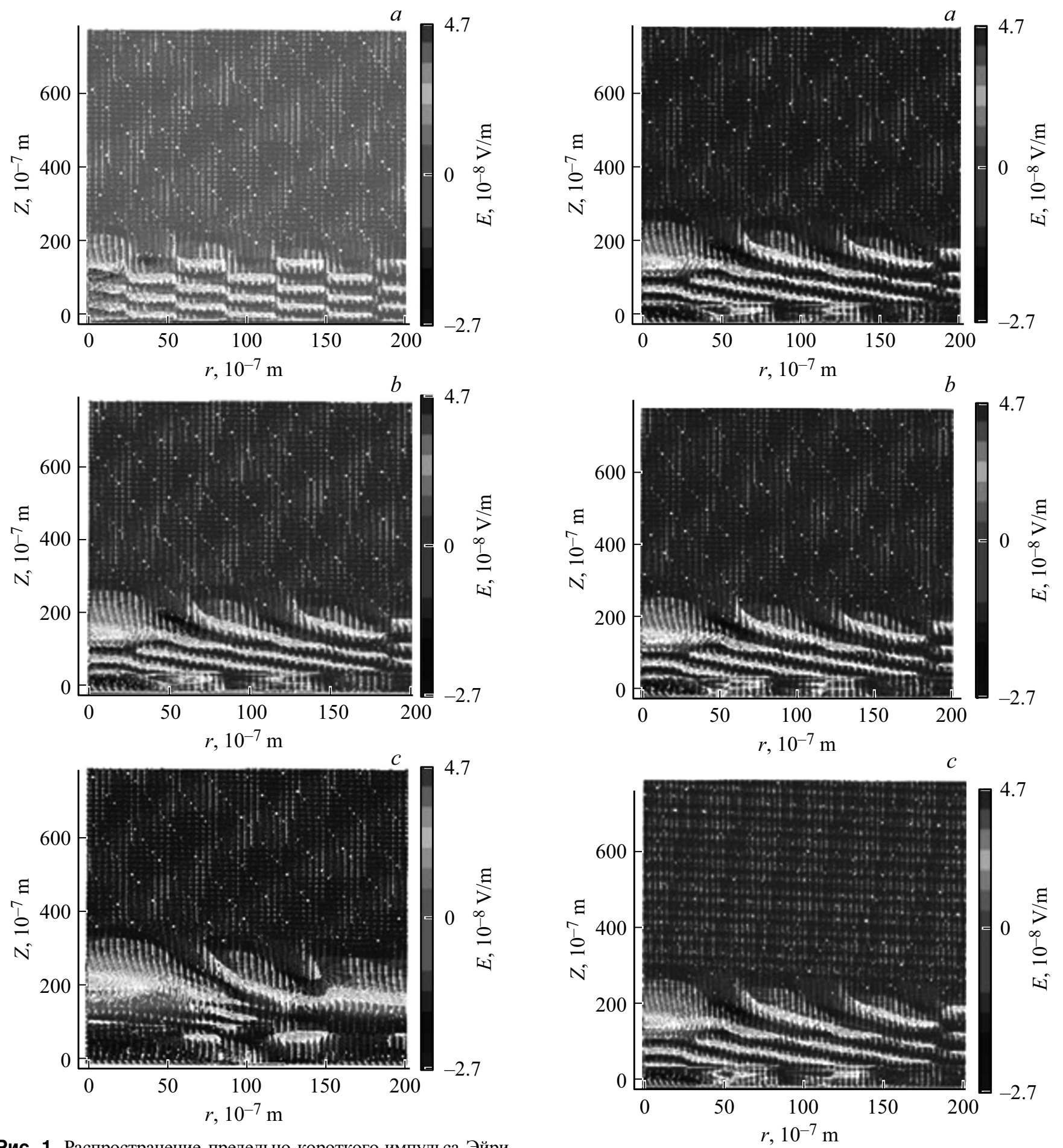

Рис. 1. Распространение предельно короткого импульса Эйри в фотонном кристалле с УНТ в различные моменты времени $t$ с фиксированными значениями глубины модуляции фотонного кристалла $\alpha=0.2$, периода фотонного кристалла $\chi=50$, параметра импульса Эйри $\kappa=0.01$ и $\gamma=0.1 . t=0.83 \cdot 10^{-12}(a)$, $5 \cdot 10^{-12}(b), 10.83 \cdot 10^{-12} \mathrm{~s}(c)$.

Здесь $Q$ - амплитуда импульса; $\gamma_{z}, \gamma_{r}$ - ширины импульса в направлении $z$ и $r$ соответственно, $u$ начальная скорость импульса, $\kappa$ - параметр импульса Эйри. Заметим, что реализация в практическом плане

таких импульсов невозможна, так как импульсы Эйри несут бесконечное количество энергии, что для практической реализации невозможно. В силу этого соответствующие импульсы были экспоненциально обрезаны, 
$\gamma$ - параметр обрезания, который вводится для того, чтобы импульс был физически реализуемым и нес конечную энергию. Под обрезанием импульса понимаем умножение на экспоненциально затухающую функцию. Параметр обрезания $\gamma$ - это характерное расстояние в поперечном направлении, на котором амплитуда импульса убывает в $e$ раз.

\section{Основные результаты}

На рис. 1 видны три разные формы импульса, они показывают как распространялся импульс с течением времени. На рис. 1, $a$ форма импульса более заостренная, нет плавных переходов по сравнению со случаями $b$ и $c$. Форма $b$ представляет собой уже плавные переходы, они более широкие и более выражены в высоту и не имеют острот. Что касается формы $c$, видно выраженные, достаточно высокие волны, которые также не имеют острот.

Как можно заметить из рис. 1, импульс Эйри распространяется устойчиво. Основная часть энергии продолжает быть сосредоточенной в центральной части импульса, хотя и наблюдается дифракционное уширение. Можно сделать вывод об устойчивости световой пули. Отметим, что в отличие от случая линейной среды в данном случае в силу нелинейности среды форма импульса уже не сохраняется.

Результат распространения импульса (рис. 2) при изменении значения глубины модуляции показателя преломления фотонного кристалла показывает, что импульс замедляется, но слабо меняет свою форму.

На рис. 3 мы отлично видим, что параметр функции Эйри $\kappa$ влияет на структуру импульса, но импульс все равно остается локализованным независимо от его значения.

Как видно из приведенных зависимостей, решение для трехмерной световой пули в фотонном кристалле остается локализованным, но меняет вследствие поперечной дисперсии свою пространственную структуру. Совместное действие эффектов расплывания импульса вследствие дисперсии и нелинейности приводит к образованию поперечной структуры, которая тем не менее остается локализованной в ограниченной пространственной области.

Проведенные расчеты показывают, что возможно устойчивое распространение трехмерных световых пуль не только в среде УНТ, но и в среде УНТ, помещенных в фотонный кристалл. Отметим, что с практической точки зрения данный результат важен тем, что позволяет управлять скоростью световых пуль, изменяя параметры фотонного кристалла. Вместе с тем распространение световых пуль в фотонном кристалле имеет и ряд важных отличий от случая среды с постоянным показателем преломления. Пожалуй, самое важное отличие состоит в том, что световые пули в фотонном кристалле имеют более сложную поперечную структуру, которая, на наш взгляд, связана с возбуждением внутренних

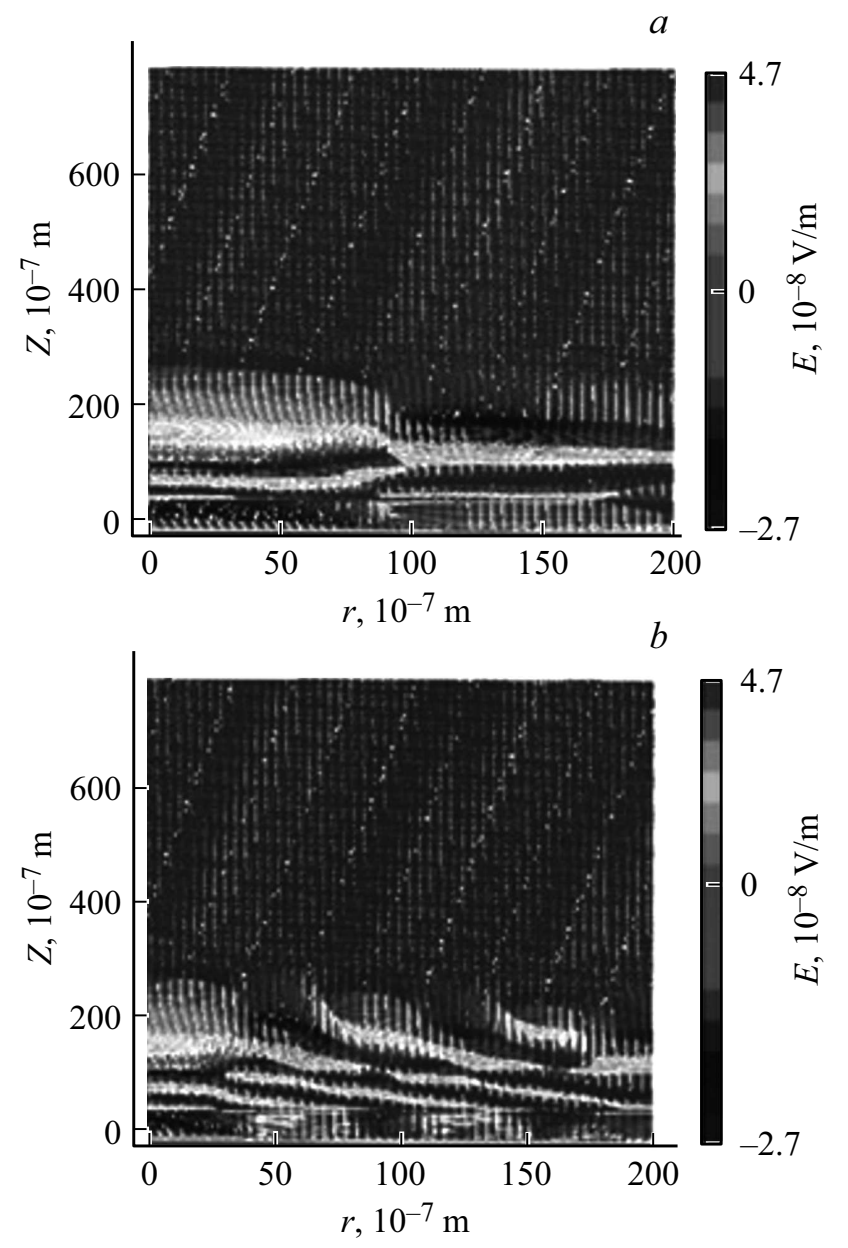

Рис. 3. Изменение формы импульса в фотонном кристалле с УНТ (с фиксированными значениями $t=5 \mathrm{ps,} \mathrm{глубины}$ модуляции фотонного кристалла $\alpha=0.2$, периода фотонного кристала $\chi=50$ и параметра импульса Эйри $\kappa=0.01)$ за счет изменения параметра импульса Эйри: $\gamma=0.03(a), 0.09(b)$.

мод колебаний световой пули при взаимодействии с неоднородностью показателя преломления среды. Ранее такой эффект уже наблюдался при решении задачи о взаимодействии предельно короткого импульса с металлической неоднородностью в среде УНТ [16].

\section{Выводы}

Из приведенного исследования можно сделать следующие выводы.

1. В фотонном кристалле с УНТ световые пули распространяются устойчиво.

2. За счет нелинейности среды форма импульса остается локализованной в пространстве и слабо изменяется.

3. Глубина модуляции показателя преломления фотонного кристалла слабо влияет на форму импульса.

4. Параметр функции Эйри влияет на структуру импульса, но форма импульса при распространении сильно не изменяется. 


\section{Список литературы}

[1] Skorobogatiy M., Yange J. Fundamentals of Photonic Crystal Guiding. Cambridge University Press, 2009. P. 11.

[2] Белоненко М.Б., Демушкина Е.В., Лебедев Н.Г. // ФТТ. 2008. T. 50. № 2. С. 368.

[3] Белоненко М.Б., Лебедев Н.Г., Попов А.С. // Письма в ЖЭТФ. 2010. Т. 91. № 9. С. 506.

[4] Zhukov A.V., Bouffanais R., Belonenko M.B., Konobeeva N.N., Nevzorova Yu.V., George T.F. // Eur. Phys. J. D. 2015. V. 69. doi 10.1140/epjd/e2015-50895-y

[5] Leblond H., Mihalache D. // Phys. Rev. A. 2012. V. 86. P. 043832. doi 10.1103/PhysRevA.86.043832

[6] Leblond H., Mihalache D. // Phys. Rep. 2013. V. 523. N 2. P. 61. doi 10.1016/j.physrep.2012.10.006

[7] Zhukov A.V., Bouffanais R.E., Fedorov G., Belonenko M.B. // J. Appl. Phys. 2013. V. 114. P. 143106. doi 10.1063/1.4824370

[8] Belonenko M.B., Nevzorova Yu.V. // Izvestiya RAN. Seriya Fizicheskaya. 2014. V. 78. P. 1619. doi $10.7868 / \mathrm{S} 0367676514120035$

[9] Belonenko M.B., Nevzorova Yu.V., Galkina E.N. // Mod. Phys. Lett. B. 2015. V. 29. P. 1550041. doi $10.1142 / \mathrm{S} 0217984915500414$

[10] Belonenko M.B., Demushkina E.V., Lebedev N.G. // Phys. Solid State. 2008. V. 50. P. 383.

[11] Durnin J., Mirceli J.J., Eberty J.H. // Phys. Rev. Lett. 1988. V. 58. N 15. P. 1499.

[12] Alexander V., Zhukov R.B., Belonenko M.B., Dvuzhilov I.S., Nevzorova Y.V. // Appl. Phys. B. 2017. V. 340. P. 195.

[13] Ананьев Ю.А. // Опт. и спектр. 1988. Т. 64. № 6. С. 1211.

[14] Belonenko M.B., Nevzorova Yu.V., Galkina E.N. // Mod. Phys. Lett. B. 2015. V. 29. P. 1550041. doi $10.1142 / \mathrm{S} 0217984915500414$

[15] Zhukov A.V., Bouffanais R., Fedorov E.G., Belonenko M.B. // J. Appl. Phys. 2013. V. 114. P. 143106.

[16] Харрис П. Углеродные нанотрубы и родственные структуры. Новые материалы XXI века. М.: Техносфера, 2003. C. 336 . 